\title{
Direct measurement of general quantum states using weak measurement
}

\author{
Jeff S. Lundeen ${ }^{1, *}$ and Charles Bamber ${ }^{1}$ \\ ${ }^{1}$ Institute for National Measurement Standards, National Research Council, 1200 Montreal Rd., Ottawa, ON, K1A 0R6
}

\begin{abstract}
Recent work [J.S. Lundeen et al. Nature, 474, 188 (2011)] directly measured the wavefunction by weakly measuring a variable followed by a normal (i.e. 'strong') measurement of the complementary variable. We generalize this method to mixed states by considering the weak measurement of various products of these observables, thereby providing the density matrix an operational definition in terms of a procedure for its direct measurement. The method only requires measurements in two bases and can be performed 'in situ', determining the quantum state without destroying it.
\end{abstract}

PACS numbers: 03.65.Ta, 03.65.Wj, 42.50.Dv, 03.67.-a

The wavefunction $\Psi$ is at the heart of quantum mechanics, yet its nature has been debated since its inception. It is typically relegated to being a calculational device for predicting measurement outcomes. Recently, Lundeen et al. proposed a simple and general operational definition of the wavefunction based on a method for its direct measurement: "it is the average result of a weak measurement of a variable followed by a strong measurement of the complementary variable [1, 2]." By 'direct' it is meant that a value proportional to the wavefunction appears straight on the measurement apparatus itself without further complicated calculations or fitting. The 'wavefunction' referred to here is a special case of a general quantum state, known as a 'pure state.' The general case is represented by the density operator $\rho$, which can describe both pure and 'mixed' states. The latter incorporates both the effects of classical randomness (e.g., noise) and entanglement with other systems (e.g., decoherence). The density operator plays an important role in quantum statistics, quantum information, and the study of decoherence. Because of its generality and because it follows naturally from classical concepts of probability and measures, some consider $\rho$, rather than $\Psi$, to be the fundamental quantum state description. In this letter, we propose two methods to directly measure general quantum states, one of which directly gives the matrix elements of $\rho$.

The standard method for experimentally determining the density operator is Quantum State Tomography [3] . In it, one makes a diverse set of measurements on an ensemble of identical systems and then determines the quantum state that is most compatible with the measurement results. An alternative is our direct measurement method, which may have advantages over tomography, such as simplicity, versatility, and directness. A quantitative comparison of measures such as the signal to noise ratio, resolution, and fidelity, has not been undertaken but some limitations of the direct method have been identified in [4]. As compared to tomography, which works with mixed states, the most significant limitation of the direct measurement of the wavefunction is that it has only been shown to work with pure states.
Previous works have developed direct methods to measure quasi-probability distributions, such as the Wigner function [5], Husimi Q-function [6], and the GlauberSudarshan P-function [7]. These are position-momentum (i.e. 'phase-space') distributions that are equivalent to the density operator, and have many, but not all, of the properties of a standard probability distribution. The Wigner function can be directly measured by displacing the system in phase space and then measuring the parity operator [8]. Equivalently, the integral of the interference between a pair of rotated and displaced replicas of the system will give the Wigner function [9]. The Husimi Q-function can be directly measured by an eight-port homodyne apparatus or by projection on the harmonic oscillator ground state [10]. These phase-space distributions are created to be the closest quantum analogs to a classical probability distribution. In this sense, they are inherently amenable to direct measurement.

Weak measurement.-We begin by considering what happens to our method for directly measuring the wavefunction when the state is not pure. At the heart the direct method is weak measurement 11]. Over the last decade, interest in weak measurement has grown as researchers have realized its potential for interrogating quantum systems in a coherent manner [12]. It has been used to model and understand photonic phenomena in birefringent photonic crystals [13], fiber networks [14], cavity QED [15], and quantum tunneling [16, 17]. Weak measurement provides insight into a number of fundamental quantum effects, including the role of the uncertainty principle in the double-slit experiment [18], the Legget-Garg inequality 19], the quantum box problem 20], and Hardy's paradox 21]. It has also been used to amplify small experimental effects 22 and as feedback for control of a quantum system [23]. Weak measurements have been demonstrated in both classical [24] and non-classical systems [25].

The concept of weak measurement is universally applicable to all types of measurement [26 28] but here we introduce it with a standard model of measurement, the von Neumann model [29]. In it, a measurement apparatus has a pointer, in an initial position wavefunction 
$\left\langle q \mid \phi_{i}\right\rangle=\phi_{i}(q) \propto \exp \left[-q^{2} /\left(4 \sigma^{2}\right)\right]$, whose momentum $\mathrm{K}$ is coupled with strength $g$ to an system observable $\mathbf{A}$ via the interaction, $\mathbf{U}=\exp (-i g \mathbf{A K} t / \hbar)$, where $t$ is the interaction duration. In a measurement of $\mathbf{A}$, the position $\mathrm{Q}$ of the pointer is shifted to indicate the result of the measurement, $\mathrm{A}=a: \phi_{i}(q) \rightarrow \phi_{f}(q)=\phi_{i}(q-a)$. In a standard (i.e.'strong', gt large) measurement, this shift is much greater than the width $\sigma$ of the pointer and, thus, unambiguously indicates the measurement result. It also leaves the system in the associated eigenstate $|a\rangle$, thereby radically disturbing it. To perform a weak measurement one reduces $g t$ such that induced pointer shift is less then $\sigma$, making the measurement result ambiguous. The benefit is that the system disturbance is reduced. While a weak measurement on a single system provides little information, by repeating it on an arbitrarily large ensemble of identical systems one can determine the $a v$ erage measurement result with arbitrary precision. We call this the 'Weak Average' $\left\langle\mathbf{A}^{\mathrm{w}}\right\rangle_{\rho}$. Unsurprisingly, it is simply equal to the standard quantum expectation value [30], $\left\langle\mathbf{A}^{\mathrm{w}}\right\rangle_{\rho}=\operatorname{Tr}[\mathbf{A} \boldsymbol{\rho}]=\left\langle\mathbf{A}^{\mathrm{s}}\right\rangle_{\rho}$, where the latter indicates $\mathbf{A}$ is measured strongly.

A distinguishing feature of weak measurement is that, in the limit of zero interaction $(g t=0)$, the quantum state of the system remains unchanged. Subsequent measurements can now provide additional information about that initial quantum state $|\Psi\rangle$. Consider a subsequent strong measurement of observable $\mathbf{C}$ that results in outcome $c$ (corresponding to eigenstate $|c\rangle$ ). The average result of the weak measurement of $\mathbf{A}$ in the sub-ensemble of systems giving $\mathrm{C}=c$ is called the 'Weak Value' and is given by [11],

$$
\left\langle\mathbf{A}^{\mathrm{w}}\right\rangle_{\Psi}^{c}=\frac{\langle c|\mathbf{A}| \Psi\rangle}{\langle c \mid \Psi\rangle}
$$

Surprisingly, the weak value can be outside the range of the eigenvalues of $\mathbf{A}$ and can even be complex 30 33]. Often consideration is limited to its real part, as would be done in standard measurement [17, 28, 30] but the imaginary part also has a physical significance: the evolution $\mathbf{U}$ not only shifts the average position of pointer but also the average momentum of the pointer. These two, purely real, shifts are proportional to the real and imaginary parts of the weak value, respectively [30 32]: $\left\langle\mathbf{A}^{\mathrm{w}}\right\rangle_{\Psi}^{c}=\langle\mathbf{Q}\rangle_{f} / g t+i\langle\mathbf{K}\rangle_{f} 2 \sigma^{2} / g t \hbar$, where $\langle\mathbf{L}\rangle_{f} \equiv\left\langle\phi_{f}|\mathbf{L}| \phi_{f}\right\rangle$. This result was generalized to other initial pointer wavefunctions [34], and discrete pointers (e.g. Qubits or Spins) 31]. The complex nature of the weak value is what enables us to directly measure the real and imaginary parts of the wavefunction and, as we show later, directly measure the Dirac distribution and density matrix.

Direct measurement of the quantum wavefunction.We now review our method for the direct measurement of the wavefunction. The concept is general, however here we consider the case of a discrete Hilbert space. In this space, one is free to choose the basis $\{|a\rangle\}$ (associated with observable $\mathbf{A}$ ) in which the wavefunction will be measured. The method consists of weakly measuring a projector in this basis $\boldsymbol{\pi}_{a} \equiv|a\rangle\langle a|$, and post-selecting on a particular value $b_{0}$ of the complementary observable B. By 'complementary' we mean that $\left\langle a \mid b_{0}\right\rangle=1 / \sqrt{N}$ for all $a$, where $N$ is the dimension of the Hilbert space. That is, the overlap is real and constant as function of $a$. The existence of state $\left|b_{0}\right\rangle$ is guaranteed by the existence of at least two mutually unbiased bases (MUB) in any Hilbert space [35]. As discussed in the supplementary information of Ref. [1, 2], the choice of state $b_{0}$ out of the basis $\{|b\rangle\}$ is simply a convention and is equivalent to choosing a reference frame for the direct measurement, thereby setting the phases of the basis states in $\{|a\rangle\}$. Using Eq. (1), the quantum state $|\Psi\rangle$ is given by: $|\Psi\rangle=$ $v \cdot \sum_{a}\left\langle\boldsymbol{\pi}_{a}^{\mathrm{w}}\right\rangle_{\Psi}^{b_{0}}|a\rangle$, where $\left\langle\boldsymbol{\pi}_{a}^{\mathrm{w}}\right\rangle_{\Psi}^{b_{0}}$ is the weak value and $v$ is a constant that is independent of $a$. Thus, by stepping through the values of $a$ in a series of weak measurements one can directly measure $|\Psi\rangle$ represented in the $a$ basis.

Weak measurement of mixed states.-The weak value of a system described by a density operator $\rho$ was first considered in [15] and shown to be:

$$
\left\langle\mathbf{A}^{\mathrm{w}}\right\rangle_{\rho}^{c}=\frac{\langle c|\mathbf{A} \boldsymbol{\rho}| c\rangle}{\langle c|\boldsymbol{\rho}| c\rangle}
$$

Applying this to our direct measurement method we find, $\left\langle\boldsymbol{\pi}_{a}^{\mathrm{W}}\right\rangle_{\rho}^{b_{0}}=\left\langle b_{0} \mid a\right\rangle\left\langle a|\boldsymbol{\rho}| b_{0}\right\rangle /\left\langle b_{0}|\boldsymbol{\rho}| b_{0}\right\rangle$. Only $2 N$ real parameters are found by scanning $a$. This will not generally be sufficient to determine all the parameters in $\rho$, which has $N^{2}-1$ real parameters. Consequently, our method for the direct measurement of the wavefunction cannot be used to determine a mixed state.

Direct measurement of the Dirac distribution.-We now consider what happens if one replaces the strong measurement of $\mathbf{B}$ with a weak measurement. Specifically, we investigate the weak measurement of the product of projectors from the two MUB, $\mathbf{S}_{a b} \equiv|b\rangle\langle b \mid a\rangle\langle a|$ with no post-selection whatsoever. We wish to measure its weak average. Although, a non-Hermitian operator $\mathbf{A}$ is typically considered to be unobservable, later, we shall outline specific methods to weakly measure it. Surprisingly, we show that even if $\mathbf{A}$ is non-Hermitian $\left\langle\mathbf{A}^{\mathrm{w}}\right\rangle_{\rho}=\operatorname{Tr}[\mathbf{A} \boldsymbol{\rho}]$ still holds for the weak average. In this case, $\left\langle\mathbf{A}^{\mathbf{w}}\right\rangle_{\rho}$ is complex with a physical significance similar to that of the weak value (i.e. shifts in the position and momentum of the pointer). For now, we use this result to find the weak average of $\mathbf{S}_{a b}$ :

$$
\left\langle\mathbf{S}_{a b}^{\mathrm{w}}\right\rangle_{\rho}=\operatorname{Tr}\left[\mathbf{S}_{a b} \boldsymbol{\rho}\right]=\langle a|\boldsymbol{\rho}| b\rangle\langle b \mid a\rangle=S_{\rho}(a, b),
$$

where $S_{\rho}(a, b)$ is the discrete Hilbert space version of the Dirac distribution as defined in [36]. Dirac introduced this phase-space distribution as a way to represent a quantum operator $\mathbf{O}$ in his 1945 paper [37], "On the 
Analogy Between Classical and Quantum Mechanics." In various guises it has been investigated periodically during last half-century 38. In optics, variations of the Dirac distribution have been used widely, appearing in Walther's definition of the radiance function in radiometry [39] and Wolf's specific intensity [40] (as pointed out in [36]). If $\mathbf{O}=\boldsymbol{\rho}$, the Dirac distribution is a representation of the quantum state of a system. For instance, the joint weak measurement of a position $x$ and a momentum $p$ (i.e. $\mathbf{S}_{x p} \equiv|p\rangle\langle p \mid x\rangle\langle x|$ ) on a mixed state $\boldsymbol{\rho}$ gives the phase-space version of the Dirac distribution, $S_{\rho}(x, p)$, which, although it is complex, shares many of the desired features of a quasi-probability distribution [36].

In our weak measurement, if one scans $a$ and $b$, so as to directly measure the Dirac distribution over all values of $(a, b)$, one completely determines the density operator. But in order to actually calculate the density operator from the Dirac distribution one must know $\langle b \mid a\rangle=\exp \left(i \theta_{a b}\right) / \sqrt{N}$. Since it is not generally known what are the bases in the MUB set (for any given Hilbert space) a general formula for $\theta_{a b}$ is also unknown. However, if $\{|a\rangle\}$ is taken to be the standard basis (i.e. $\sum_{a=0}^{N}|a\rangle\langle a|=\mathbf{I}$, the identity operator) then one MUB, which we take to be $\{|b\rangle\}$, will always be the Fourier basis [35, 41],$|b\rangle=\sum_{a=0}^{N-1}|a\rangle \exp (i 2 \pi a b / N) / \sqrt{N}$. In this case, $\theta_{a b}=-2 \pi a b / N$, where $a$ and $b$ are integers solely used to enumerate the states such that $0 \leq$ $a, b \leq N-1$. With these choices for our complementary bases the density operator is simply related to the Dirac distribution by a Discrete Fourier Transform, $\rho_{a_{1} a_{2}}=$ $\sum_{b=0}^{N-1} S_{\rho}\left(a_{1}, b\right) e^{i 2 \pi b\left(a_{1}-a_{2}\right) / N}$, where $\rho_{a_{1} a_{2}}=\left\langle a_{1}|\boldsymbol{\rho}| a_{2}\right\rangle$. This explicitly shows the weak average, $\left\langle\mathbf{S}_{a b}^{\mathrm{w}}\right\rangle_{\rho}$, contains the same information as the density operator. Much like the Wigner function, the Dirac distribution can used to find the expectation value of an observable through a simple overlap integral [36]. Unlike the Wigner function, it is compatible with Bayes' law and, thus, is consistent with a quantum analog of classical determinism [42].

Direct measurement of the density operator.-While quasi-probability distributions are informationally equivalent to the density operator, they are less commonly known and used. Motivated by a desire to understand the nature of the density operator we now describe how to measure it directly in a given basis. Consider the weak measurement of the product of three projectors, $\boldsymbol{\Pi}_{a_{1} a_{2}}=$ $\boldsymbol{\pi}_{a_{2}} \boldsymbol{\pi}_{b_{0}} \boldsymbol{\pi}_{a_{1}}$, where $\boldsymbol{\pi}_{a_{1}}=\left|a_{1}\right\rangle\left\langle a_{1}\left|, \boldsymbol{\pi}_{b_{0}}=\right| b_{0}\right\rangle\left\langle b_{0}\right|$, and $\boldsymbol{\pi}_{a_{2}}=\left|a_{2}\right\rangle\left\langle a_{2}\right|$ and $b_{0}$ is chosen so that $\left\langle a \mid b_{0}\right\rangle=1 / \sqrt{N}$ for all $a$ (but is not required to be from the Fourier basis). As before, there is no post-selection. The weak average is:

$$
\left\langle\boldsymbol{\Pi}_{a_{1} a_{2}}^{\mathrm{w}}\right\rangle_{\rho}=\left\langle a_{1}|\boldsymbol{\rho}| a_{2}\right\rangle / N=\rho_{a_{1} a_{2}} / N .
$$

Thus, each element $\rho_{a_{1} a_{2}}$ of the density matrix in any chosen basis (here $\{|a\rangle\}$ ) can be measured directly by weakly measuring the corresponding projectors, $\boldsymbol{\pi}_{a_{1}}$ and $\pi_{a_{2}}$, in between which is a third weak measurement of $\pi_{b_{0}}$. The proportionality constant $N^{-1}$ can be eliminated through the normalization of the density matrix so that $\operatorname{Tr}[\boldsymbol{\rho}]=1$. Keeping $b_{0}$ fixed while scanning $a_{1}$ and $a_{2}$ allows one to map out the entire density matrix.

Weak measurement of products of complementary variables.- One cause for concern in our two direct measurement methods is that $\mathbf{S}_{a b}$ and $\boldsymbol{\Pi}_{a_{1} a_{2}}$ are not Hermitian, which, according to the postulates of quantum mechanics, means they are not observable [43]. Indeed, coupling such operators to a pointer via the von Neumann interaction (as in $\mathbf{U}$ ) leads to an unphysical non-unitary evolution. This issue can be circumvented by dividing the measurement into a sequence of unitary von Neumann interactions. Each has a pointer beginning in same initial state $\phi_{i}(q)$. We now describe a pair of schemes that use this strategy to weakly measure the product of two non-commuting observables $\mathbf{E}$ and $\mathbf{F}$ thereby measuring their weak average $\left\langle(\mathbf{E F})^{\mathrm{w}}\right\rangle_{\rho}$. In the process, we will show that the weak average $\left\langle(\mathbf{E F})^{\mathrm{w}}\right\rangle_{\rho}=\operatorname{Tr}[\mathbf{E F} \boldsymbol{\rho}]$. And later we will show that weakly measuring just two observables is sufficient to implement both direct measurement methods.

Scheme 1: The first scheme follows a commonly used strategy for standard (strong) measurements: perform independent measurements of two observables and correlate the results to find the observables' product. With von Neumann measurements the total evolution is $\mathbf{U}_{T} \equiv$ $\exp \left(i g_{2} \mathbf{E K}_{2} t / \hbar\right) \exp \left(i g_{1} \mathbf{F} \mathbf{K}_{1} t / \hbar\right)$, where the subscripts indicate observables on pointer 1 or 2 . If $\mathbf{E}$ and $\mathbf{F}$ commute, strong measurements give $\left\langle(\mathbf{E F})^{\mathrm{s}}\right\rangle_{\rho} \propto\left\langle\mathbf{Q}_{1} \mathbf{Q}_{2}\right\rangle_{f}$. The weak measurement version of this strategy was proposed in [44]. It was simplified in [31] by forming the composite operator $\mathfrak{a} \equiv \mathbf{Q} / 2 \sigma+i \mathbf{K} \sigma / \hbar$, which has the form of an annihilation operator (i.e. $\mathfrak{a}\left|\phi_{i}\right\rangle=0$ ), so that the standard weak value (Eq. 1) has the simple form $\left\langle\mathbf{A}^{\mathrm{w}}\right\rangle_{\Psi}^{c}=(2 \sigma / g t)\langle\mathfrak{a}\rangle_{f}$. Following [31], one can show that in the limit $g_{1} g_{2}(t / \sigma)^{2} \ll 1$, the evolution $\mathbf{U}_{T}$ induces the pointer shifts $\left\langle(\mathbf{E F})^{\mathrm{w}}\right\rangle_{\rho}=\operatorname{Tr}[\mathbf{E F} \boldsymbol{\rho}]=$ $g_{1} g_{2}(2 \sigma / t)^{2}\left\langle\mathfrak{a}_{1} \mathfrak{a}_{2}\right\rangle_{f}$. This scheme was demonstrated experimentally in [21] for products of commuting observables. Ref. [34] showed that it is valid even for noncommuting observables $\mathbf{E}$ and $\mathbf{F}$ if they are measured sequentially, as in $\mathbf{U}_{T}$. (This result can be generalized to an $n$-product observable, such as the triple product $\boldsymbol{\Pi}_{a_{1} a_{2}}$ 31, 34].) Thus, just as with strong measurement, by performing independent measurements of each observable and then evaluating a joint expectation value on the pointers one can measure $\left\langle(\mathbf{E F})^{\mathrm{w}}\right\rangle_{\rho}$.

Scheme 2: The second scheme measures $\mathbf{F}$ and then, conditioned on the result, measures $\mathbf{E}$, thereby measuring their product. A von Neumann interaction couples $\mathbf{F}$ to a first pointer, which shifts the pointer's position. With a strength proportional to 
this shift a second von Neumann interaction couples $\mathbf{E}$ to a second pointer. This conditional sequential measurement is described by the total interaction $\mathbf{U}_{\mathrm{D}} \equiv \exp \left(-i g_{2} \mathbf{E} \mathbf{K}_{2} \mathbf{Q}_{1} t / \hbar\right) \exp \left(-i g_{\mathrm{D}} \mathbf{F} \mathbf{D}_{1} t / \hbar\right)$, where the rightmost interaction couples to either $\mathbf{D}=\mathbf{Q}$ or $\mathbf{K}$, and the subscripts refer to pointer 1 or 2 . For $\mathbf{D}=\mathbf{K}$ our weak measurement of $\mathbf{F}$ is a standard von Neumann interaction. In the limit of $g_{K} g_{2} t^{2} / \sigma \ll 1$, the evolution $\mathbf{U}_{\mathrm{K}}$ shifts the position of pointer 2 by $\left\langle\mathbf{Q}_{2}\right\rangle_{f}=\left(g_{K} g_{2} t^{2}\right) \operatorname{Re}\{\operatorname{Tr}[\mathbf{E F} \boldsymbol{\rho}]\}$. However, $\left\langle\mathbf{K}_{2}\right\rangle_{f}=0$, leaving us without $\operatorname{Im}\{\operatorname{Tr}[\mathbf{E F} \boldsymbol{\rho}]\}$. The imaginary component can be found by coupling $\mathbf{F}$ to the position, rather than momentum, of the first pointer. If we set $\mathbf{D}=\mathbf{Q}$, then in the limit $g_{Q} g_{2} t^{2} \sigma / \hbar \ll 1$, the evolution $\mathbf{U}_{\mathrm{Q}}$ shifts the momentum of the second pointer by $\left\langle\mathbf{Q}_{2}\right\rangle_{f}=\left(2 g_{\mathrm{Q}} g_{2} t^{2} \sigma^{2} / \hbar\right) \operatorname{Im}\{\operatorname{Tr}[\mathbf{E F} \boldsymbol{\rho}]\}$. In summary, conditional two sequential weak measurements allow us to measure the real and imaginary components of expectation value of a product operator.

Both of the schemes can be followed by post-selection of some other observable and, in that case, they would give the weak value of $\mathbf{E F}$, which can be complex. However, our schemes show that, even without post-selection, the weak average of a non-Hermitian $\mathbf{E F}$ will be complex. This may come as a surprise since post-selection is often cited as the mechanism for anomalous weak values [22, 24, 30].

How to substitute a strong measurement for a weak measurement of one observable in a product.-We now show that the weak measurement of $\mathbf{C G}$ is equivalent to first weakly measuring $\mathbf{G}$ followed by a strong measurement of $\mathbf{C},\left\langle(\mathbf{C G})^{\mathrm{w}}\right\rangle=\left\langle\mathbf{C}^{\mathrm{s}} \mathbf{G}^{\mathrm{w}}\right\rangle$. This can greatly simplify both of our proposed methods. In analogy to a standard joint expecation value, by $\left\langle\mathbf{C}^{\mathrm{s}} \mathbf{G}^{\mathrm{w}}\right\rangle$ we mean that for each measured outcome of the strong measurement of $\mathbf{C}$ one multiplies the corresponding eigenvalue $c$ by its probability $P(c)$ by the weak value $\left\langle\mathbf{G}^{\mathbf{w}}\right\rangle_{\rho}^{c}$ to find the average, $\sum_{c} c P(c)\left\langle\mathbf{G}^{\mathrm{w}}\right\rangle_{\rho}^{c} \equiv\left\langle\mathbf{C}^{\mathrm{s}} \mathbf{G}^{\mathrm{w}}\right\rangle$. Using Eq. (2), it follows that, $\left\langle\mathbf{C}^{\mathbf{s}} \mathbf{G}^{\mathbf{w}}\right\rangle=\sum_{c} c\langle c|\mathbf{G} \boldsymbol{\rho}| c\rangle=\operatorname{Tr}[\mathbf{C G} \boldsymbol{\rho}]=$ $\left\langle(\mathbf{C G})^{\mathrm{w}}\right\rangle$. In other words, a joint weak-strong measurement of $\mathbf{G}$ and $\mathbf{C}$, respectively, will have the average result, $\operatorname{Tr}[\mathbf{C G} \boldsymbol{\rho}]$. Note that because both the weak value and corresponding the pointer expectation values (e.g. $\left.\langle\mathbf{Q}\rangle_{f}\right)$ are normalized by $P(c)$, in an actual experiment the pointer signal will be proportional to $\langle c|\mathbf{G} \boldsymbol{\rho}| c\rangle$ directly, removing the need to find the weak value.

Consequently, we can directly measure the full Dirac distribution and density matrix by measuring the correlations between the weak measurement and the subsequent strong measurement outcome while we scan the weak measurement. For the density matrix one would weakly measure $\boldsymbol{\pi}_{b_{0}} \boldsymbol{\pi}_{a_{1}}$ using scheme 1 or 2 and then strongly measure of $\boldsymbol{\pi}_{a_{2}}$. Alternatively, one can instead strongly measure $\mathbf{A}$, which projects on the eigenstates $\left\{\boldsymbol{\pi}_{a}\right\}$ in parallel. This has the advantage that the final measurement no longer must be scanned. Similarly, in the experiment in [1], replacing the slit with a camera (with a preceding polarization analyzer) in the momentum plane is the only modification necessary to directly measure the Dirac distribution for the transverse density matrix of a photon. Evidently, dividing the joint measurement into weak and strong parts simplifies each of the two new direct measurements considered in this paper (given in Eq. (3) and Eq. (4)) and makes them feasible with existing technology (e.g. weak measurement of the product of two operators [21]).

Conclusion.-In this work, we have shown that by weakly measuring pairs or triple products of complementary variables of a system it is possible not only to directly measure its wavefunction but, also, its density operator. To measure over the extent of wavefunction we only need to scan the first variable of a complementary pair. To determine the density operator through its Dirac distribution we must, additionally, scan the second variable. To determine the density matrix directly, one weakly measures the product of a variable, its complementary variable, and the first variable again. Leaving the complementary variable fixed at one value while rastering the values of the other two completely maps the density matrix, one element at a time. This procedure thus provides the density matrix with an operational definition, it is the average result of a joint weak measurement of a variable, then its complementary variable, then the original variable.

These methods also provide alternatives to standard state tomography that have three key advantages: One, they are simpler in that they only require measurements in two of the system's bases. Two, they do not require a global reconstruction - states can be determined locally, point by point. And three, the amount of state disturbance can be minimized. Thus, in principle, we can characterize quantum states in situ, for instance, in the middle of quantum computation circuits, or during chemical reactions, without disturbing the process in which they feature.

We thank Aephraim Steinberg for useful discussions.

* jeff.lundeen@nrc-cnrc.gc.ca

[1] J. S. Lundeen, B. Sutherland, A. Patel, C. Stewart, and C. Bamber, Nature 474, 188 (2011).

[2] J. S. Lundeen, Generalized Measurement and Postselection in Optical Quantum Information, Ph.D. thesis, University of Toronto (2006).

[3] K. Vogel and H. Risken, Phys. Rev. A 40, 2847 (1989), D. T. Smithey, M. Beck, M. G. Raymer, and A. Faridani, Phys. Rev. Lett. 70, 1244 (1993) G. Breitenbach, S. Schiller, and J. Mlynek, Nature 387, 471 (1997); A. G. White, D. F. V. James, P. H. Eberhard, and P. G. Kwiat, Phys. Rev. Lett. 83, 3103 (1999).

[4] E. Haapasalo, P. Lahti, and J. Schultz, Phys. Rev. A 84, 052107 (2011). 
[5] E. P. Wigner, Phys. Rev. 40, 749 (1932).

[6] K. Husimi, Proc. Phys.-Math. Soc. Japan 22, 264 (1940).

[7] R. J. Glauber, Phys. Rev. 131, 2766 (1963); E. C. G. Sudarshan, Phys. Rev. Lett. 10, 277 (1963).

[8] K. Banaszek and K. Wódkiewicz, Phys. Rev. Lett. 76, 4344 (1996) K. Laiho, K. N. Cassemiro, D. Gross, and C. Silberhorn, Phys. Rev. Lett. 105, 253603 (2010).

[9] E. Mukamel, K. Banaszek, I. A. Walmsley, and C. Dorrer, Opt. Lett. 28, 1317 (2003); B. J. Smith, B. Killett, M. G. Raymer, I. A. Walmsley, and K. Banaszek, ibid. 30, 3365 (2005).

[10] U. Leonhardt, Measuring the quantum state of light, Cambridge studies in modern optics (Cambridge University Press, 1997); J. F. Kanem, S. Maneshi, S. H. Myrskog, and A. M. Steinberg, J. Opt. B 7, S705 (2005).

[11] Y. Aharonov, D. Z. Albert, and L. Vaidman, Phys. Rev. Lett. 60, 1351 (1988).

[12] Y. Aharonov, S. Popescu, and J. Tollaksen, Phys. Today 63, $27(2010)$ A. Cho, Science 333, 690 (2011)

[13] D. R. Solli, C. F. McCormick, R. Y. Chiao, S. Popescu, and J. M. Hickmann, Phys. Rev. Lett. 92, 043601 (2004).

[14] N. Brunner, A. Acin, D. Collins, N. Gisin, and V. Scarani, Phys. Rev. Lett. 91, 180402 (2003); N. Brunner, V. Scarani, M. Wegmuller, M. Legre, and N. Gisin, ibid. 93, 203902 (2004).

[15] H. M. Wiseman, Phys. Rev. A 65, 032111 (2002).

[16] A. M. Steinberg, Phys. Rev. Lett. 74, 2405 (1995).

[17] A. M. Steinberg, Phys. Rev. A 52, 32 (1995).

[18] H. M. Wiseman, Phys. Lett. A 311, 285 (2003); R. Mir, J. S. Lundeen, M. W. Mitchell, A. M. Steinberg, J. L. Garretson, and H. M. Wiseman, New J. Phys. 9, 287 (2007).

[19] N. S. Williams and A. N. Jordan, Phys. Rev. Lett. 100, 026804 (2008); A. Palacios-Laloy, F. Mallet, F. Nguyen, P. Bertet, D. Vion, D. Esteve, and A. N. Korotkov, Nature Phys. 6, 442 (2010), M. E. Goggin, M. P. Almeida, M. Barbieri, B. P. Lanyon, J. L. O'Brien, A. G. White, and G. J. Pryde, Proc. Natl. Acad. Sci. U.S.A. 108, 1256 (2011).

[20] K. J. Resch, J. S. Lundeen, and A. M. Steinberg, Phys. Lett. A 324, 125 (2004).

[21] J. S. Lundeen and A. M. Steinberg, Phys. Rev. Lett. 102, 020404 (2009).

[22] O. Hosten and P. Kwiat, Science 319, 787 (2008);
P. B. Dixon, D. J. Starling, A. N. Jordan, and J. C. Howell, Phys. Rev. Lett. 102, 173601 (2009) A. Feizpour, X. Xing, and A. M. Steinberg, Phys. Rev. Lett. 107, 133603 (2011)

[23] G. A. Smith, S. Chaudhury, A. Silberfarb, I. H. Deutsch, and P. S. Jessen, Phys. Rev. Lett. 93, 163602 (2004) G. G. Gillett, R. B. Dalton, B. P. Lanyon, M. P. Almeida, M. Barbieri, G. J. Pryde, J. L. O'Brien, K. J. Resch, S. D. Bartlett, and A. G. White, Phys. Rev. Lett. 104, 080503 (2010)

[24] N. W. M. Ritchie, J. G. Story, and R. G. Hulet, Phys. Rev. Lett. 66, 1107 (1991).

[25] G. J. Pryde, J. L. O'Brien, A. G. White, T. C. Ralph, and H. M. Wiseman, Phys. Rev. Lett. 94, 220405 (2005).

[26] O. Oreshkov and T. A. Brun, Phys. Rev. Lett. 95, 110409 (2005).

[27] H. F. Hofmann, Phys. Rev. A 81, 012103 (2010)

[28] J. Dressel, S. Agarwal, and A. N. Jordan, Phys. Rev. Lett. 104, 240401 (2010).

[29] J. von Neumann, Mathematical Foundations of Quantum Mechanics (Princeton Univ. Press, 1955).

[30] Y. Aharonov and L. Vaidman, Phys. Rev. A 41, 11 (1990).

[31] J. S. Lundeen and K. J. Resch, Phys. Lett. A 334, 337 (2005).

[32] R. Jozsa, Phys. Rev. A 76, 044103 (2007).

[33] H. F. Hofmann, New Journal of Physics 13, 103009 (2011).

[34] G. Mitchison, Phys. Rev. A 77, 052102 (2008)

[35] T. Durt, B. Englert, I. Bengtsson, and K. Zyczkowski, Int. J. Quantum Inf. 8, 535 (2010).

[36] S. Chaturvedi, E. Ercolessi, G. Marmo, G. Morandi, N. Mukunda, and R. Simon, J. Phys. A 39, 1405 (2006).

[37] P. A. M. Dirac, Rev. Mod. Phys. 17, 195 (1945)

[38] L. M. Johansen, Phys. Rev. A 76, 012119 (2007)

[39] A. Walther, J. Opt. Soc. Am. 58, 1256 (1968)

[40] E. Wolf, Phys. Rev. D 13, 869 (1976).

[41] I. Bengtsson, in Foundations of Probability and Physics - 4 . American Institute of Physics Conference Series, Vol. 889, edited by G. Adenier, C. Fuchs, \& A. Y. Khrennikov (2007) pp. 40-51.

[42] H. F. Hofmann, ArXiv e-prints (2011), arXiv:1107.0536 [quant-ph]

[43] R. Shankar, Principles of quantum mechanics (Plenum Press, 1994).

[44] K. J. Resch and A. M. Steinberg, Phys. Rev. Lett. 92, 130402 (2004). 